\title{
MIMO Detection for High Order QAM by Canonical Dual Approach
}

\author{
Ye $\operatorname{Tian}^{1}$ and Jr-Fong Dang ${ }^{2}$ \\ ${ }^{1}$ School of Business Administration, Southwestern University of Finance and Economics, Chengdu 611130, China \\ ${ }^{2}$ Department of Industrial Engineering \& Management, National Chiao Tung University, Hsinchu 300, Taiwan \\ Correspondence should be addressed to Jr-Fong Dang; jrfongdang@gmail.com
}

Received 25 October 2014; Accepted 23 March 2015

Academic Editor: Panayotis Takis Mathiopouloss

Copyright (c) 2015 Y. Tian and J.-F. Dang. This is an open access article distributed under the Creative Commons Attribution License, which permits unrestricted use, distribution, and reproduction in any medium, provided the original work is properly cited.

\begin{abstract}
We develop a canonical dual approach for solving the MIMO problem. First, a special linear transformation is introduced to reformulate the original problem into a $\{-1,1\}$ constrained quadratic programming problem. Then, we derive a canonical dual problem which is piecewise continuous problem with no duality gap. Under certain conditions, the canonical problem becomes a concave maximization dual problem over a convex feasible domain. By getting the stationary point of the canonical dual problem, we can find either an optimal or approximate solution of the original problem. A gradient decent algorithm is proposed to solve the MIMO problem and simulation results are provided to demonstrate the effectiveness of the method.
\end{abstract}

\section{Introduction}

In the recent decade, multiple antennae communication systems have developed very fast since it could provide substantial performance gain over their single antenna counterparts [1]. Therefore, how to efficiently detect the signal vector of transmitted symbols becomes an important issue. As a consequent result, the problem of multiple-input-multiple-output (MIMO) detection has been raised up and received considerable attention over several years.

Note that, in the communication scenarios, the signal model is always written in the following form:

$$
y=H x+v,
$$

where $y \in \mathbb{R}^{m}$ is a received signal vector, $x \in \mathbb{R}^{n}$ is a transmitted symbol vector whose elements are drawn from a symbol constellation set, $H$ is $m \times n$ real matrix that characterizes the input-output relation, and $v \in \mathbb{R}^{m}$ is an additive white Gaussian noise with unit variance. It is worth pointing out that the signal model is sometimes represented in a complexvalued form. But it is easy to reformulate the complex-valued model to a real-valued model (please see [2] for details). The MIMO problem aims to detect the transmitted vector $x$ based on the observations $y$ and $H$. And the optimal symbol vector which minimizes the error probability can be found by solving the maximum-likelihood (ML) detection problem [3]. It is a very fundamental problem in the communication area. The corresponding problem is as follows:

$$
\begin{array}{ll}
\min & \|y-H x\|^{2} \\
\text { s.t. } & x_{i} \in\{ \pm 1, \pm 3, \ldots, \pm u\}, \quad i=1, \ldots, n,
\end{array}
$$

where $\|\cdot\|$ denotes the 2 -norm and each symbol $x_{i}$ is drawn from a $(u+1)$-QAM constellation set $S=\{ \pm 1, \pm 3, \ldots, \pm u\}$ (please see [4] for details). In this paper, we suppose $u \geq 3$. Unfortunately, the MIMO problem is NP-hard [5]. Therefore, researchers have developed a lot of approximation algorithms.

Lattice decoding is an important research direction for the MIMO detection. It has received a lot of attention for its good tradeoff between detection accuracy and complexity [6$8]$. Naive lattice decoding (NLD) method relaxes the symbol bound constraints and finds the closest lattice point to the received signal over the whole lattice generated by the channel [6]. In order to further improve the efficiency, some suboptimal lattice decoding methods, such as sampled decoding [9], embedded decoding [10], and lattice reduction-aided (LRA) 
methods [11], can be combined with lattice decoding method to accelerate the lattice point search. However, since the NLD method completely ignores the symbol bounds, it fails to achieve the optimal diversity-multiplexing tradeoff (DMT) under general MIMO system models $[6,8]$. Then, in order to prevent the lattice points going too far away from the origin point, some researchers developed the regularized lattice decoding (RLD) method which adds a quadratic penalization term to the lattice decoding metric [12]. Though the RLD method has been empirically found to be computationally fast for small to moderate problems sizes, its complexity would be prohibitive for large $n$ and higher order QAM [13].

Another big family of MIMO detection algorithms is based on semidefinite relaxation (SDR). The SDR method relaxes the ML detection problem into a convex semidefinite programming (SDP) problem which leads to a polynomialtime complexity in the problem dimension. The SDR detector was first developed for the binary phase-shift keying (BPSK) constellation [14] and then extended to QPSK (4-QAM) constellation [15]. Researchers have verified that the SDR detector can provide a constant factor approximation to the optimal log-likelihood value in the low signal-to-noise ration (SNR) region almost surely [16]. Based on that, Wiesel et al. [17] proposed a polynomial-inspired SDR (PI-SDR) method for 16-QAM and proved that PI-SDR achieves an optimal Lagrangian dual lower bound of the ML. Sidiropoulos and Luo [18] designed a bound-constrained SDR (BC-SDR) method which has a special structure. Thus, compared to PISDR, BC-SDR makes fast implementations more favorable. Moreover, Mao et al. [19] developed a virtually antipodal SDR (VA-SDR) method for any $4^{q}$-QAM (where $q>1$ ). For the relationship and comparisons between these SDR detectors, please see [2]. Though SDP problem has a theoretical low polynomial computational complexity, due to large problem size and slow SDP solvers, the actual computation time is very high in practice.

Besides, there are some other algorithms for the MIMO problem. Sphere decoder method is a classical one $[4,20]$. However, it exhibits exponential complexity with respect to the problem size. Moreover, Goldberger and Leshem [21] proposed a new detection algorithm based on an optimal tree approximation in an unconstrained linear system. They showed that this algorithm outperforms other methods for the loop-free factor graph situation. Recently, Pan et al. [22] proposed a Lagrangian dual relaxation (LDR) for the MIMO problem. This method finds the best diagonally regularized lattice decoder to approximate the ML detector. They proved that the LDR problem yields a duality gap no worse than that of the SDR method.

In this paper, we present a canonical duality approach to the MIMO problem. The canonical duality theory is originally proposed for handling general nonconvex and/or nonsmooth systems [23]. Canonical dual transformation and associated triality theory play a key role in the implementation. It is worth pointing out that the canonical dual transformation may convert a nonconvex and/or nonsmooth primal problem into a piecewise smooth canonical dual problem. And this dual problem has no duality gap in each subregion. Therefore, this powerful tool has a big potential in some global optimization problems and nonconvex nonsmooth analysis [24-28]. Particularly, the canonical duality theory can be applied to the quadratic programming problem with integer constraints. Fang et al. [29] proposed a more general global optimization condition using the canonical duality approach. Wang et al. [30] developed a canonical duality approach for solving multi-integer quadratic programming problems. Thus, this paper adopts the canonical duality approach to study the MIMO problem.

The paper is arranged as follows. In Section 2, we introduce a special linear transformation to reformulate the original problem into a $\{-1,1\}$ constrained quadratic programming problem. In Section 3, we develop the canonical dual problem for the new reformulation. Then we show that it has no duality gap under certain conditions. Moreover, some global optimality conditions are presented. In Section 4, we propose a gradient decent method to find the stationary points. Comparisons by simulations are provided in Section 5. The last section summarizes the paper and points out some future research directions.

\section{Linear Transformation}

The MIMO problem can be written as follows:

$$
\begin{array}{ll}
\min & x^{T}\left(H^{T} H\right) x-2 y^{T} H x+\|y\|^{2} \\
\text { s.t. } & x_{i} \in\{ \pm 1, \pm 3, \ldots, \pm u\}, \quad i=1, \ldots, n .
\end{array}
$$

Let $Q=H^{T} H$ and $f=2 H^{T} y$. Note that $Q$ is $n \times n$ real symmetric matrix and $f$ is $n$-dimensional real vector. Since $\|y\|^{2}$ is a fixed scalar, problem (MIMO1) has the same optimal solutions with the following problem:

$$
\begin{array}{ll}
\min & x^{T} Q x-f^{T} x \\
\text { s.t. } & x_{i} \in\{ \pm 1, \pm 3, \ldots, \pm u\}, \quad i=1, \ldots, n .
\end{array}
$$

We first reformulate problem (MIMO2) into a $\{-1,1\}$ constrained quadratic programming problem. Note that the set $\{ \pm 1, \pm 3, \ldots, \pm u\}$ forms an arithmetic series; that is, the adjacent two elements in the set have a constant gap 2 . Therefore, we can take advantage of this special structure.

Let $y_{i}=\left(x_{i}+u\right) / 2$ for $i=1, \ldots, n$; then $y_{i} \in\{0,1, \ldots, u\}$. Let $e$ denote the $n$-dimensional vector with all elements being 1 . Then, it is easy to verify that problem (MIMO2) is equivalent to the following problem:

$$
\begin{array}{ll}
\min & 4 y^{T} Q y-\left(4 u e^{T} Q+2 f^{T}\right) y+u^{2} e^{T} Q e+u f^{T} e \\
\text { s.t. } & y_{i} \in\{0,1, \ldots, u\}, \quad i=1, \ldots, n .
\end{array}
$$

(MIMO3)

Problem (MIMO2) and problem (MIMO3) have the same optimal value. Moreover, once we get an optimal solution of problem (MIMO3), we can use $x=2 y-u e$ to get the corresponding optimal solution of problem (MIMO2). Now, let $\bar{Q}=4 Q$ and $\bar{f}=4 u Q^{T} e+2 f$. Since $u^{2} e^{T} Q e+u f^{T} e$ is 
also a fixed scalar, problem (MIMO3) has the same optimal solutions with the following problem:

$$
\begin{array}{ll}
\min & y^{T} \bar{Q} y-\bar{f}^{T} y \\
\text { s.t. } & y_{i} \in\{0,1, \ldots, u\}, \quad i=1, \ldots, n .
\end{array}
$$

(MIMO4)

Let $Y=\left\{y \in \mathbb{R}^{n} \mid y_{i}\right.$ is an integer, $\left.0 \leq y_{i} \leq u\right\}$ denote the feasible domain of problem (MIMO4). Set $t=\lfloor\log (u+1)\rfloor$; then $t \geq 2$ and $u+1 \geq 2^{t}$. Now, we can define a new set $Z$ as follows:

$$
\mathscr{Z}=\left\{z \in \mathbb{R}^{(t+1) n} \mid z \in\{-1,1\}^{(t+1) n}\right\} .
$$

Note that each element in set $Z$ is a $(t+1) n$-dimensional vector with all elements being -1 or 1 . Then, we have the following theorem to show the relationship between $Y$ and $Z$.

Theorem 1. The transformation $y_{i}(z)=\sum_{j=1}^{t} 2^{j-2} z_{(j-1) n+i}+$ $(1 / 2)\left(u+1-2^{t}\right) z_{t n+i}+u / 2$ is a full mapping from $\mathscr{Z}$ to $Y$.

Proof. We first show that, for any $z \in Z$, the corresponding $y$ belongs to $Y$. Note that $y_{i}(z)$ can also be written as $y_{i}(z)=$ $\sum_{j=2}^{t} 2^{j-2} z_{(j-1) n+i}-2^{t-1} z_{t n+i}+(1 / 2) z_{i}+((u+1) / 2) z_{t n+i}+u / 2$. The first part $\sum_{j=2}^{t} 2^{j-2} z_{(j-1) n+i}-2^{t-1} z_{t n+i}$ is always an integer; the possible fraction can only occur in the second part $(1 / 2) z_{i}+$ $((u+1) / 2) z_{t n+i}+u / 2$. However, it is easy to verify that this part is always an integer as $z_{i}, z_{t n+i} \in\{-1,1\}$ for $u \geq 3, t \geq 2$. Moreover, for $i=1, \ldots, n, \max \left\{y_{i}\right\}=\sum_{j=1}^{t} 2^{j-2}+\left(u+1-2^{t}\right) / 2+$ $u / 2=u$ and $\min \left\{y_{i}\right\}=-\sum_{j=1}^{t} 2^{j-2}-\left(u+1-2^{t}\right) / 2+u / 2=0$. Therefore, $y_{i}$ is an integer between 0 and $u$ and $y$ belongs to $Y$.
Then, we show that, for an arbitrary integer $0 \leq y_{i} \leq u$, there exist $z_{(j-1) n+i} \in-1,1$ for $j=1, \ldots, t+1$ such that the transformation holds. If $0 \leq y_{i} \leq 2^{t}-1$, then $y_{i}$ can be written as $y_{i}=2^{t-1} a_{t-1}+2^{t-2} a_{t-2}+\cdots+2 a_{1}+1 a_{0}$ for particular $a_{i} \in\{0,1\}$ and $j=0, \ldots, t-1$. Then, let $z_{j n+i}=2 a_{j}-1$ for $j=0, \ldots, t-1$ and $y_{t n+i}=-1$. Thus, $z_{(j-1) n+i} \in\{-1,1\}$ for $j=1, \ldots, t+1$. It is easy to verify that $\sum_{j=1}^{t} 2^{j-2} z_{(j-1) n+i}+$ $\left(\left(u+1-2^{t}\right) / 2\right) z_{t n+i}+u / 2=\sum_{j=0}^{t-1} 2^{j} a_{j}=y_{i}$. If $2^{t}-1<y_{i} \leq u$, since $2^{t} \leq u+1 \leq 2^{t+1}$, we have $-2^{t} \leq 2^{t}-(u+1) \leq 0$. Therefore, $0 \leq y_{i}+2^{t}-(u+1) \leq 2^{t}-1$. Then following the similar way, we can write $y_{i}+2^{t}-(u+1)$ as $y_{i}+2^{t}-(u+1)=$ $2^{t-1} a_{t-1}+2^{t-2} a_{t-2}+\cdots+2 a_{1}+1 a_{0}$ for particular $a_{i} \in 0,1$, $j=0, \ldots, t-1$. This time, let $z_{j n+i}=2 a_{j}-1$ for $j=0, \ldots, t-1$ and $z_{t n+i}=1$. Thus, $z_{(j-1) n+i} \in\{-1,1\}$ for $j=1, \ldots, t+1$. Consequently, we have $\sum_{j=1}^{t} 2^{j-2} z_{(j-1) n+i}+\left(\left(u+1-2^{t}\right) / 2\right) z_{t n+i}+$ $u / 2=\sum_{j=0}^{t-1} 2^{j} a_{j}+(u+1)-2^{t}=x_{i}$. $Y$.

Therefore, the transformation is a full mapping from $Z$ to

It is worth pointing out that this transformation is a linear mapping. Moreover, since we use the advantage of the special structure of the original problem, the size of the reformulated problem is smaller than the problems derived by some traditional transformation methods [3].

Now, replacing $y$ by $z$ in problem (MIMO4), we can get the following problem:

$$
\begin{array}{ll}
\min & z^{T} M z-b^{T} z+c \\
\text { s.t. } & z_{i} \in\{-1,1\}, \quad i=1, \ldots,(t+1) n,
\end{array}
$$

where

$$
M=\left(\begin{array}{ccccc}
\frac{1}{4} \bar{Q} & \begin{array}{c}
\frac{1}{2} \bar{Q} \\
\frac{1}{2} \bar{Q}
\end{array} & \cdots & 2^{t-3} \bar{Q} & \frac{1}{4}\left(u+1-2^{t}\right) \bar{Q} \\
\vdots & \bar{Q} & \cdots & 2^{t-2} \bar{Q} & \frac{1}{2}\left(u+1-2^{t}\right) \bar{Q} \\
2^{t-3} \bar{Q} & \vdots & \ddots & \vdots \\
\frac{1}{4}\left(u+1-2^{t}\right) \bar{Q} & \frac{1}{2}\left(u+1-2^{t}\right) \bar{Q} & \cdots & 2^{t-3}\left(u+1-2^{t}\right) \bar{Q} & \frac{1}{4}\left(u+1-2^{t}\right)^{2} \bar{Q}
\end{array}\right),
$$


Therefore, from Theorem 1, solving problem (MIMO5) is equivalent to solving problem (MIMO4).

Let $r=(t+1) n$ and ignore the fixed scalar $c$; we focus on the following problem:

$$
\begin{array}{ll}
\min & F(z)=z^{T} M z-b^{T} z \\
\text { s.t. } & z_{i} \in\{-1,1\}, \quad i=1, \ldots, r .
\end{array}
$$

Note that problem (MIMO6) has no linear constraints and its feasible domain is merely defined by $Z=\left\{z \in \mathbb{R}^{r}\right.$ | $\left.z \in\{-1,1\}^{r}\right\}$.

\section{Canonical Dual Problem and Global Optimality}

Let $\rho \in \mathbb{R}^{r}$ be a vector and let $\operatorname{Diag}(\rho)$ denote an $r \times r$ diagonal matrix with $\rho_{i}$ being the $i_{\text {th }}$ diagonal element. Let

$$
\begin{gathered}
M_{\rho}=M+\operatorname{Diag}(\rho), \\
P(\rho)=-\frac{1}{4} b^{T}\left(M_{\rho}\right)^{-1} b-e^{T} \rho .
\end{gathered}
$$

Moreover, we define a set as follows:

$$
\mathscr{D}_{M}=\left\{\rho \in \mathbb{R}^{r}|\rho \geq 0,| M_{\rho} \mid \neq 0\right\},
$$

where $\left|M_{\rho}\right|$ is the determinant of the matrix $M_{\rho}$.

Then following the work of $[30,31]$, we can get the canonical dual problem of problem (MIMO6) as follows:

$$
\operatorname{sta}\left\{P(\rho) \mid \rho \in \mathscr{D}_{M}\right\} \text {, }
$$

$(\mathrm{CDP})$

where $e$ is an $r$-dimensional vector with all elements being 1 . $\operatorname{sta}\left\{P(\rho) \mid \rho \in \mathscr{D}_{M}\right\}$ represents finding all stationary points (critical points) of $P(\rho)$ over $\mathscr{D}_{M}$.

Moreover, for two vectors $a, b \in \mathbb{R}^{n}$, let $a \circ b$ denote the standard Hadamard product $a \circ b \triangleq\left(a_{1} b_{1}, a_{2} b_{2}, \ldots, a_{r} b_{r}\right)^{T}$. Then, for the relationship between the primal problem and the dual problem, we have the next two important theorems.

Theorem 2. If $\rho^{*} \in \mathscr{D}_{M}$ is a stationary point of the dual objective function $P(\rho)$, then $z\left(\rho^{*}\right)=(1 / 2)\left(M_{\rho^{*}}\right)^{-1} b$ is an integer vector in $\{-1,1\}^{r}$. Moreover, if $M_{\rho^{*}}$ is positive definite, then the Hessian matrix of $P\left(\rho^{*}\right)$ is negative definite.

Proof. Note that since $z=(1 / 2)\left(M_{\rho^{*}}\right)^{-1} b$, we have $M_{\rho^{*}} z=$ $(1 / 2) b$. Thus, $\left.\left(d\left(M_{\rho} z\right) / d \rho\right)\right|_{\rho=\rho^{*}}=(d(M z+\operatorname{Diag}(\rho) z) /$ $d \rho)\left.\right|_{\rho=\rho^{*}}=\left.M(d z / d \rho)\right|_{\rho=\rho^{*}}+\operatorname{Diag}(z)+\left.\operatorname{Diag}\left(\rho^{*}\right)(d z / d \rho)\right|_{\rho=\rho^{*}}$ $=0$. Hence, we have $\left.(d z / d \rho)\right|_{\rho=\rho^{*}}=-\left(M_{\rho^{*}}\right)^{-1} \operatorname{Diag}(z)$. Therefore, $\left.(d P(\rho) / d \rho)\right|_{\rho=\rho^{*}}=-(1 / 2)\left(d\left[\left(b^{T}\left(M_{\rho}\right)^{-1} M_{\rho} z\right)+\right.\right.$ $\left.\left.2 e^{T} \rho\right] / d \rho\right)\left.\right|_{\rho=\rho^{*}}=-\left.(1 / 2)\left(d\left(b^{T} z\right) / d \rho\right)\right|_{\rho=\rho^{*}}-e=-(1 / 2)(d z /$ $d \rho)\left.^{T}\right|_{\rho=\rho^{*}} b-e=(1 / 2) \operatorname{Diag}(z)\left(M_{\rho^{*}}\right)^{-1} b-e=\operatorname{Diag}(z) z-e=$ $z \circ z-e$. Therefore, if $\rho^{*}$ is a stationary point of $P(\rho)$, then $z\left(\rho^{*}\right) \circ z\left(\rho^{*}\right)-e=0$. This implies that the vector $z\left(\rho^{*}\right)=$ $(1 / 2)\left(M_{\rho^{*}}\right)^{-1} b \in\{-1,1\}^{r}$.
Moreover, the Hessian matrix $\left.\left(d^{2} P(\rho) / d(\rho)^{2}\right)\right|_{\rho=\rho^{*}}=$ $\left.(d(z \circ z-e) / d \rho)\right|_{\rho=\rho^{*}}=\left.2 \operatorname{Diag}(z)(d z / d \rho)\right|_{\rho=\rho^{*}}=$ $-2 \operatorname{Diag}(z)\left(M_{\rho^{*}}\right)^{-1} \operatorname{Diag}(z)$. Thus, if $M_{\rho^{*}}$ is positive definite, then the Hessian matrix of $P\left(\rho^{*}\right)$ is negative definite.

Theorem 2 indicates that a stationary point $\rho^{*}$ of $P(\rho)$ over $\mathscr{D}_{M}$ is corresponding to a feasible solution of the primal problem (MIMO6).

Theorem 3. The canonical dual problem (CDP) is perfectly dual to the primal problem (MIMO6) in the sense that if $\bar{\rho} \epsilon$ $\mathscr{D}_{M}$ is a stationary point of $P(\rho)$, then $\bar{z}=(1 / 2)\left(M_{\bar{\rho}}\right)^{-1} b$ is a KKT point of problem (MIMO6) and $F(\bar{z})=P(\bar{\rho})$.

Proof. Note that problem (MIMO6) can be written as follows:

$$
\begin{array}{ll}
\min & F(z)=z^{T} M z-b^{T} z \\
\text { s.t. } & z \circ z-e=0,
\end{array}
$$

$$
z \in \mathbb{R}^{r}
$$

Its corresponding Lagrangian function is

$$
L(z, \rho)=z^{T} M z-b^{T} z+(\rho)^{T}(z \circ z-e) .
$$

Therefore, the KKT conditions are as follows:

$$
\begin{aligned}
& \frac{\partial L}{\partial z}=2 M z-b+2 \operatorname{Diag}(\rho) z=2 M_{\rho} z-b=0, \\
& \frac{\partial L}{\partial \rho}=z \circ z-e=0 .
\end{aligned}
$$

Thus, from Theorem 2 and the definition of $\bar{z}(\bar{\rho})$, it is easy to verify that $\bar{z}(\bar{\rho})$ is a KKT point if $\bar{\rho}$ is a stationary point of $P(\rho)$.

Moreover, we have

$$
\begin{aligned}
\bar{z}^{T} M \bar{z} & -b^{T} \bar{z} \\
& =\bar{z}^{T}(M+\operatorname{Diag}(\bar{\rho})) \bar{z}-b^{T} \bar{z}-\bar{z}^{T} \operatorname{Diag}(\bar{\rho}) \bar{z} \\
& =\frac{1}{2} \bar{z}^{T} b-b^{T} \bar{z}-\bar{z}^{T} \operatorname{Diag}(\bar{\rho}) \bar{z} \\
& =-\frac{1}{2} b^{T} \bar{z}-\bar{z}^{T} \operatorname{Diag}(\bar{\rho}) \bar{z} \\
& =-\frac{1}{2} b^{T} \bar{z}-e^{T} \bar{\rho} \\
& =-\frac{1}{4} b^{T}\left(M_{\bar{\rho}}\right)^{-1} b-e^{T} \bar{\rho} \\
& =P(\bar{\rho}) .
\end{aligned}
$$

The result follows.

KKT conditions provide necessary conditions for local minimizers in a nonconvex programming problem. Next, we 
show that the canonical dual problem is a concave maximization dual problem over a convex feasible domain under certain conditions. First, we define a subset of set $\mathscr{D}_{M}$ as follows:

$$
\mathscr{D}_{M}^{+}=\left\{\rho \in \mathbb{R}^{r} \mid \rho \geq 0, M_{\rho}>0\right\},
$$

where $M_{\rho^{*}}>0$ indicates that $M_{\rho^{*}}$ is positive definite. Then, we present the sufficient global optimal conditions.

Theorem 4. Assume that $\bar{\rho}^{*} \in \mathscr{D}_{M}$ is a stationary point of $P(\rho)$ and $\bar{z}\left(\bar{\rho}^{*}\right)=(1 / 2)\left(M_{\bar{\rho}^{*}}\right)^{-1} b$. If $\bar{\rho}^{*} \in \mathscr{D}_{M}^{+}$, then $\bar{z}$ is a unique global minimizer of $F(z)$ over $Z$ and $\bar{\rho}^{*}$ is a global maximizer of $P\left(\bar{\rho}^{*}\right)$ over $\mathscr{D}_{M}^{+}$with

$$
\bar{z}^{T} M \bar{z}-b^{T} \bar{z}=\min _{z \in Z} z^{T} M z-b^{T} z=\max _{\rho^{*} \in \mathscr{D}_{M}^{+}} C\left(\rho^{*}\right)=C\left(\bar{\rho}^{*}\right) .
$$

Proof. Note that if $\rho \in \mathscr{D}_{M}^{+}$, then $M_{\rho}>0$. Thus, Theorem 2 indicates that the Hessian matrix of $P(\rho)$ is negative definite over $\mathscr{D}_{M}^{+}$. Then we know that the canonical dual function $P(\rho)$ is strictly concave over $\mathscr{D}_{M}^{+}$. Therefore, a stationary point of $P(\rho)$ must be a global maximizer of $P(\rho)$ over $\mathscr{D}_{M}^{+}$. Since the stationary point $\bar{\rho}^{*} \in \mathscr{D}_{M}^{+}$, Theorem 3 indicates that $F(\bar{z})=P\left(\bar{\rho}^{*}\right)$.

On the other hand, since $\bar{z}$ is a KKT point assured by Theorem 3, we have $\bar{z} \circ \bar{z}-e=0$. Therefore, $F(\bar{z})=$ $\bar{z}^{T} M \bar{z}-b^{T} \bar{z}+\left(\bar{\rho}^{*}\right)^{T}(\bar{z} \circ \bar{z}-e)=\bar{z}^{T}\left(Q+\operatorname{Diag}\left(\bar{\rho}^{*}\right)\right) \bar{z}-b^{T} \bar{z}-$ $e^{T} \bar{\rho}^{*}$. Note that $Q+\operatorname{Diag}\left(\bar{\rho}^{*}\right)$ is positive definite; therefore $\bar{z}=(1 / 2)\left(M_{\bar{\rho}^{*}}\right)^{-1} b$ is a global minimizer. Therefore, $\bar{z}^{T}(Q+$ $\left.\operatorname{Diag}\left(\bar{\rho}^{*}\right)\right) \bar{z}-b^{T} \bar{z}-e^{T} \bar{\rho}^{*}=\min _{z \in \mathbb{R}^{r}}\left\{z^{T}\left(Q+\operatorname{Diag}\left(\bar{\rho}^{*}\right)\right) z-b^{T} z-\right.$ $\left.e^{T} \bar{\rho}^{*}\right\} \leq \min _{\left\{z \in \mathbb{R}^{r}, z \circ z=e\right\}} z^{T} Q z-b^{T} z$. Therefore, $\bar{z}$ is a global minimizer of $F(z)$ over $Z$.

Above all, we transform the original problem which is a multi-integer quadratic programming problem into a piecewise continuous canonical dual problem by using the canonical duality theory. It is worth pointing out that the original problem and the canonical dual problem have no duality gap over $\mathscr{D}_{M}$. Moreover, if $\rho^{*} \in \mathscr{D}_{M}^{+}$, the canonical dual problem becomes a concave maximization problem over a convex feasible domain which can be solved very efficiently. To the best of our knowledge, among all the existing methods, strong duality only holds for the Lagrangian dual relaxation (LDR) in the special 2-PAM case [22]. However, the canonical dual problem holds for any order PAM problem over $\mathscr{D}_{M}$. Therefore, in some sense, the canonical dual problem provides the best dual problem of all.

\section{Algorithm}

Note that if $\rho^{*}$ is a stationary point of $P(\rho)$ over $\mathscr{D}_{M}$, then $z\left(\rho^{*}\right)=(1 / 2)\left(M_{\rho^{*}}\right)^{-1} b$ is a feasible solution of problem (MIMO6). Moreover, if $\rho^{*} \in \mathscr{D}_{M}^{+}$, the corresponding solution $z\left(\rho^{*}\right)$ is the global optimal solution of problem (MIMO6). Therefore, the solution of the dual problem (CDP) is corresponding to an optimal or approximation solution of problem (MIMO6).

The key issue for solving the problem (CDP) is to avoid the matrix $M_{\rho^{*}}$ becoming a singular matrix. Since $z\left(\rho^{*}\right)=$ $(1 / 2)\left(M_{\rho^{*}}\right)^{-1} b$, we have $2\left(M+\operatorname{Diag}\left(\rho^{*}\right)\right) z\left(\rho^{*}\right)=b$. Thus, $2 \operatorname{Diag}\left(z\left(\rho^{*}\right)\right) \rho^{*}=b-2 M z\left(\rho^{*}\right)$. Note that $(\operatorname{Diag}(z))^{-1}=$ $\operatorname{Diag}(z)$ for $z \in Z$. Therefore, $\rho^{*}=(1 / 2)\left(\operatorname{Diag}\left(z\left(\rho^{*}\right)\right) b-\right.$ $\left.2 \operatorname{Diag}\left(z\left(\rho^{*}\right)\right) M z\left(\rho^{*}\right)\right)$. Hence, $\rho^{*}=(1 / 2)\left(z\left(\rho^{*}\right) \circ b-\right.$ $\left.2\left(M z\left(\rho^{*}\right)\right) \circ z\left(\rho^{*}\right)\right)$. Therefore, if the matrix $M_{\rho^{*}}$ is close to a singular matrix, we can perturb the vector $b$ to change the value of $\rho^{*}$ and avoid the situation.

Let $\|z\|_{\infty}$ denote the infinity norm of vector $z$. Then, we propose an algorithm based on the gradient methods to solve problem (CDP).

\section{Canonical Dual Algorithm}

Step 1 (initialization step). Let $T=\max _{i=1}^{n} \sum_{j=1}^{n} M_{i j}, \epsilon>0$ be a sufficiently small number. Let $k=0, \rho^{0}=4(T+\epsilon) e$ be the initial feasible solution of problem (CDP).

Step 2. Let $z\left(\rho^{k}\right)=(1 / 2)\left(M_{\rho^{*}}\right)^{-1} b$. If $\left\|\nabla P\left(\rho^{k}\right)\right\|_{\infty} \leq \epsilon$, go to Step 4. Otherwise, $\rho^{k+1}=\rho^{k}-\alpha^{k} g^{k}$, where $\alpha^{k}=\left\|\nabla P\left(\rho^{k}\right)\right\|_{\infty}$ is a nonnegative scalar and $g^{k}=\nabla P\left(\rho^{k}\right) ; k=k+1$.

Step 3. If $\left|M_{\rho^{k}}\right|<\epsilon$, then let $b=\left(1+1 /\|b\|_{\infty}\right) b$ and $\rho^{k}=$ $(1 / 2)\left(z\left(\rho^{k}\right) \circ b-2\left(M z\left(\rho^{k}\right)\right) \circ z\left(\rho^{k}\right)\right)$. Go back to Step 2 .

Step 4. Return $z\left(\rho^{k}\right)$ and use Theorem 1 to get the corresponding solution $y^{*}$.

\section{Comparisons by Simulations}

In this section, we use simulations to compare the canonical dual method (CDM) with some other benchmarked approximating methods, such as inexact ML sphere decoding (ML-SD) [4], semidefinite relaxation (SDR) [18], and inexact MMSE lattice decoding (MMSE-LD) [11].

The channel matrix $H$ comprises i.i.d. elements drawn from a zero-mean normal distribution of unit variance. The symbol vector $x$ is element-wise i.i.d. uniformly distributed with each element $s_{i}$ drawn from the standard $(u+1)^{2}$-QAM constellation set. Moreover, $v$ is the additive white Gaussian noise with zero mean and variance $\sigma_{v}^{2}$. Note that the signal-tonoise ratio (SNR) is defined as $E\left(\|H x\|^{2}\right) / E\left(\|v\|^{2}\right)=n\left(\sigma_{x}^{2} / \sigma_{v}^{2}\right)$, where $\sigma_{x}^{2}$ is the variance of the elements of $x$.

We use two different problem sizes as $\left(m_{1}, n_{1}\right)=(8,8)$ and $\left(m_{2}, n_{2}\right)=(16,16)$. And for each case, we test every numerical example under two situations: 16-QAM and 64QAM. All the simulations are implemented using MATLAB 7.9.0 on a computer with Intel Core $2 \mathrm{CPU} 2.50 \mathrm{Ghz}$ and $2 \mathrm{G}$ memory. Moreover, the solvers of cvx [32] are incorporated in solving the SDP problems.

Figures 1 and 2 plot the SERs of the various methods versus SNR under different problem sizes for 16-QAM constellations and 64-QAM constellations, respectively. 

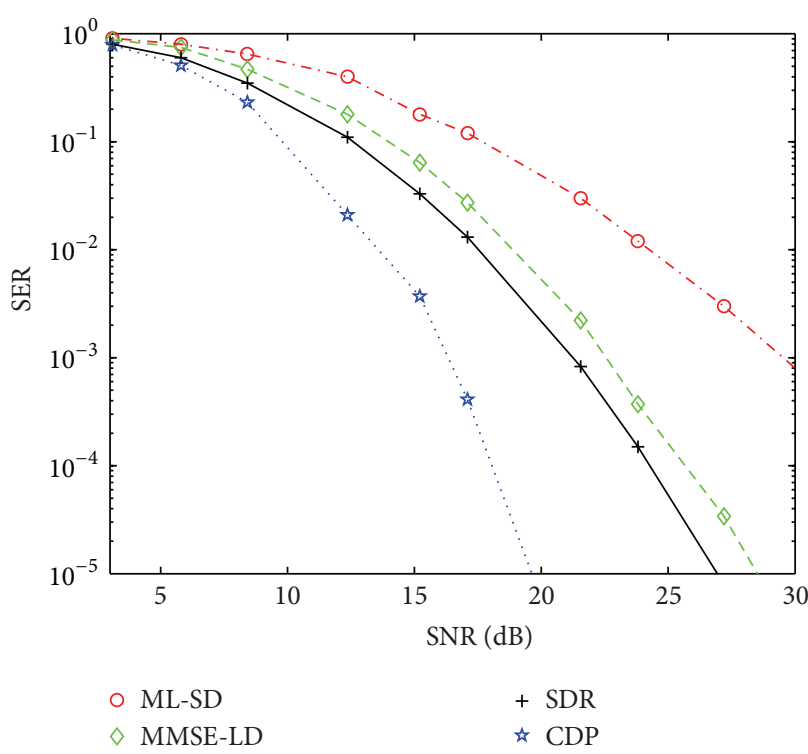

(a) 16-QAM

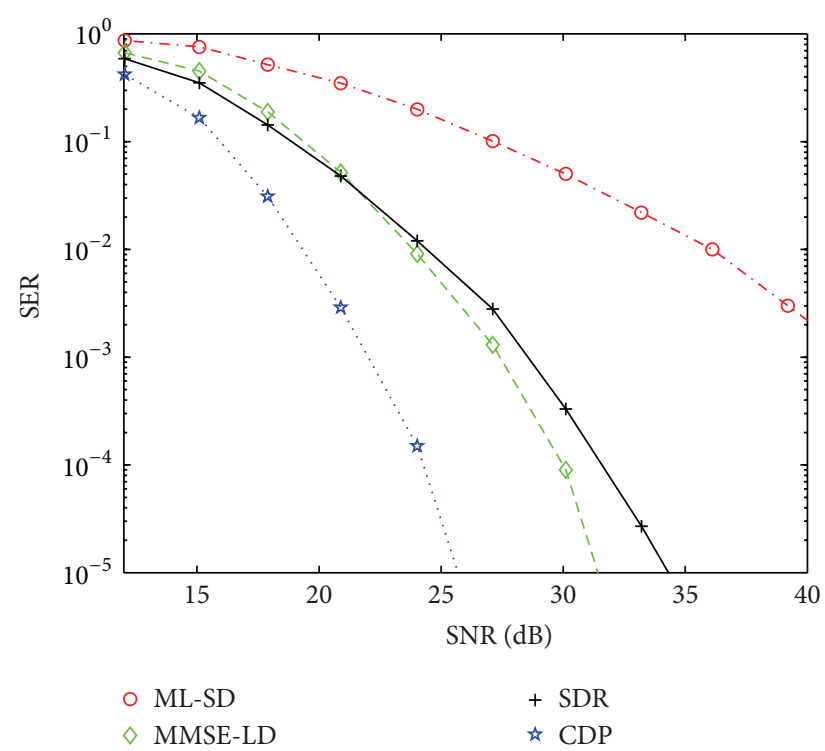

(b) 64-QAM

FIGURE 1: Symbol error rate comparison for different methods, $(m, n)=(8,8)$.

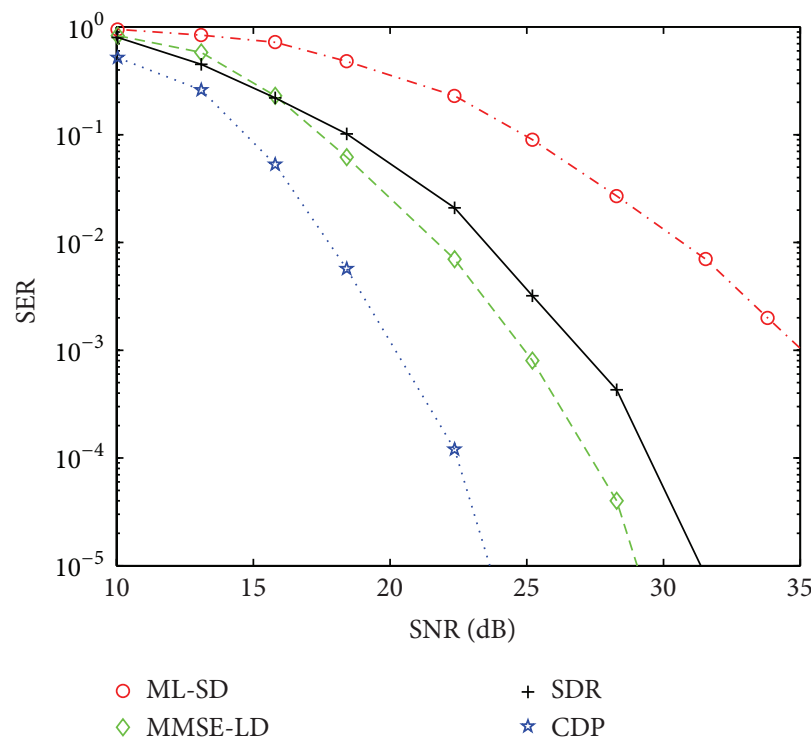

(a) 16-QAM

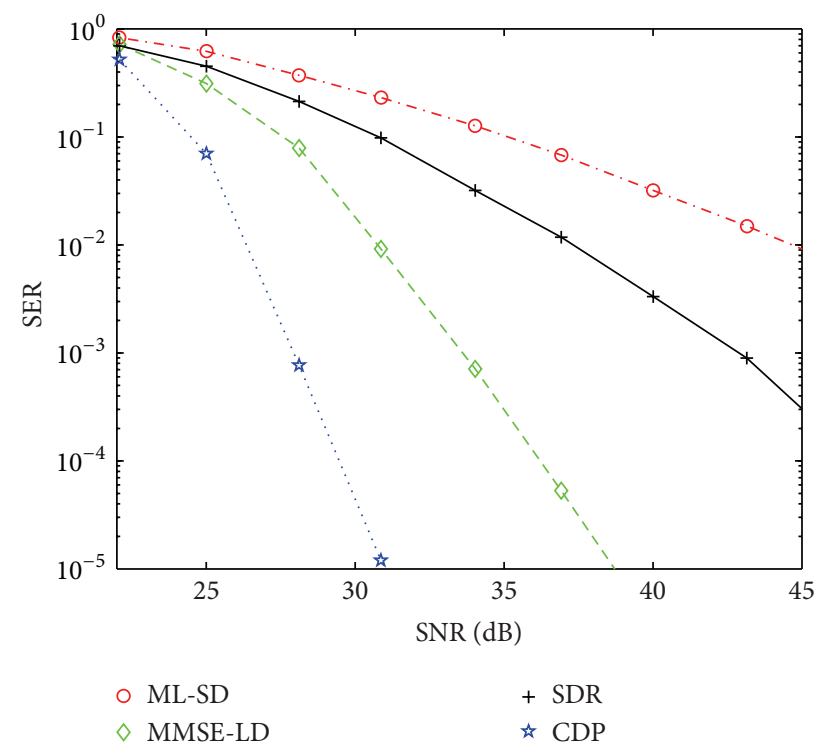

(b) 64-QAM

FIgURE 2: Symbol error rate comparison for different methods, $(m, n)=(16,16)$.

Table 1 provides the average computational time in seconds for each method under different cases. Note that the notation "8 16-QAM" denotes the 16-QAM problem whose size is $(m, n)=(8,8)$. The other notations follow the same way.

From the simulation results, we can see that the CDM method outperforms all other methods in all situations. And the SER gaps between the CDM method and other methods are significantly wide. Moreover, the computation time indicates that the CDM method is quite efficient compared with other methods. It is worth pointing out that the CDM method is much more effective for the high order QAM problem.

\section{Conclusion}

In this paper, we have developed a canonical dual method to solve the MIMO problem. By introducing a tricky linear transformation, the original problem can be reformulated as a $\{-1,1\}$ constrained quadratic programming problem with the smallest size. Then, a canonical dual problem has been developed. The stationary points of the canonical dual problem correspond to the KKT points of the original problem. And there is no duality gap between the primal problem and its canonical dual problem under some conditions. Solving the canonical dual problem can lead to either an exact or 
TABLE 1: Average computational time for different methods under different cases.

\begin{tabular}{lcccc}
\hline \multirow{2}{*}{ Methods } & \multicolumn{4}{c}{ Cases } \\
& 816-QAM & 16 16-QAM & $864-\mathrm{Q} A M$ & $1664-\mathrm{Q} A M$ \\
\hline ML-SD & $8.04 \mathrm{~s}$ & $31.2 \mathrm{~s}$ & $15.39 \mathrm{~s}$ & $38.44 \mathrm{~s}$ \\
SDR & $6.21 \mathrm{~s}$ & $28.17 \mathrm{~s}$ & $10.32 \mathrm{~s}$ & $36.45 \mathrm{~s}$ \\
MMSE-LD & $10.42 \mathrm{~s}$ & $35.76 \mathrm{~s}$ & $18.47 \mathrm{~s}$ & $54.53 \mathrm{~s}$ \\
CDM & $7.27 \mathrm{~s}$ & $36.89 \mathrm{~s}$ & $14.23 \mathrm{~s}$ & $45.17 \mathrm{~s}$ \\
\hline
\end{tabular}

approximate solution of the original problem. The simulation results indicate that the canonical dual method performs quite well compared with other benchmark methods.

The canonical dual approach offers a different angle to study the MIMO problem. It sheds some light on designing a new but more efficient algorithm. For the future study, some searching methods can be combined in the algorithm to improve the efficiency for finding the stationary points. And we aim to figure out which subclass of the MIMO problem can be exactly solved by the canonical duality theory.

\section{Conflict of Interests}

The authors declare that there is no conflict of interests related to this paper.

\section{Acknowledgment}

Ye Tian's research has been supported by the Chinese National Science Foundation no. 11401485.

\section{References}

[1] D. Tse and P. Viswanath, Fundamentals of Wireless Communication, Cambridge University Press, Cambridge, UK, 2005.

[2] W.-K. Ma, C.-C. Su, J. Jalden, T.-H. Chang, and C.-Y. Chi, “The equivalence of semidefinite relaxation MIMO detectors for higher-order QAM," IEEE Journal on Selected Topics in Signal Processing, vol. 3, no. 6, pp. 1038-1052, 2009.

[3] S. Verdú, Multiuser Detection, Cambridge University Press, Cambridge, UK, 1998.

[4] M. O. Damen, H. El Gamal, and G. Caire, "On maximumlikelihood detection and the search for the closest lattice point," IEEE Transactions on Information Theory, vol. 49, no. 10, pp. 2389-2402, 2003.

[5] S. Verdú, "Computational complexity of optimum multiuser detection," Algorithmica, vol. 4, no. 3, pp. 303-312, 1989.

[6] H. El Gamal, G. Caire, and M. Damen, "Lattice coding and decoding achieve the optimal diversity-multiplexing tradeoff of MIMO channels," IEEE Transactions on Information Theory, vol. 50, no. 6, pp. 968-985, 2004.

[7] A. K. Singh, P. Elia, and J. Jaldèn, "Achieving a vanishing SNR gap to exact lattice decoding at a subexponential complexity," IEEE Transactions on Information Theory, vol. 58, no. 6, pp. 3692-3707, 2012.

[8] M. Taherzadeh and A. K. Khandani, "On the limitations of the naive lattice decoding," IEEE Transactions on Information Theory, vol. 56, no. 10, pp. 4820-4826, 2010.
[9] S. Liu, C. Ling, and D. Stehlè, "Decoding by sampling: a randomized lattice algorithm for bounded distance decoding," IEEE Transactions on Information Theory, vol. 57, no. 9, pp. 5933-5945, 2011.

[10] L. Luzzi, D. Stehlè, and C. Ling, "Decoding by embedding: correct decoding radius and DMT optimality," IEEE Transactions on Information Theory, vol. 59, no. 5, pp. 2960-2973, 2013.

[11] D. Wübben, D. Seethaler, J. Jaldèn, and G. Matz, "Lattice reduction,” IEEE Signal Processing Magazine, vol. 28, no. 3, pp. 70-91, 2011.

[12] J. Jaldèn and P. Elia, "DMT optimality of LR-aided linear decoders for a general class of channels, lattice designs, and system models," IEEE Transactions on Information Theory, vol. 56, no. 10, pp. 4765-4780, 2010.

[13] J. Jaldèn and B. Ottersten, "On the complexity of sphere decoding in digital communications," IEEE Transactions on Signal Processing, vol. 53, no. 4, pp. 1474-1484, 2005.

[14] P. H. Tan and L. K. Rasmussen, "The application of semidefinite programming for detection in CDMA," IEEE Journal on Selected Areas in Communications, vol. 19, no. 8, pp. 1442-1449, 2001.

[15] W.-K. Ma, T. N. Davidson, K. M. Wong, and P.-C. Ching, "A block alternating likelihood maximization approach to multiuser detection," IEEE Transactions on Signal Processing, vol. 52, no. 9, pp. 2600-2611, 2004.

[16] M. Kisialiou and Z.-Q. Luo, "Performance analysis of quasimaximum-likelihood detector based on semi-definite programming," in Proceedings of the IEEE International Conference on Acoustics, Speech, and Signal Processing (ICASSP '05), vol. 3, pp. III433-III436, March 2005.

[17] A. Wiesel, Y. C. Eldar, and S. Shamai, "Semidefinite relaxation for detection of 16-QAM signaling in MIMO channels," IEEE Signal Processing Letters, vol. 12, no. 9, pp. 653-656, 2005.

[18] N. D. Sidiropoulos and Z.-Q. Luo, "A semidefinite relaxation approach to MIMO detection for high-order QAM constellations," IEEE Signal Processing Letters, vol. 13, no. 9, pp. 525-528, 2006.

[19] Z. Mao, X. Wang, and X. Wang, "Semidefinite programming relaxation approach for multiuser detection of QAM signals," IEEE Transactions on Wireless Communications, vol. 6, no. 12, pp. 4275-4279, 2007.

[20] E. Viterbo and J. Boutros, "A universal lattice code decoder for fading channels," IEEE Transactions on Information Theory, vol. 45, no. 5, pp. 1639-1642, 1999.

[21] J. Goldberger and A. Leshem, "MIMO detection for high-order QAM based on a Gaussian tree approximation," IEEE Transactions on Information Theory, vol. 57, no. 8, pp. 4973-4982, 2011.

[22] J. Pan, W.-K. Ma, and J. Jaldèn, "MIMO detection by Lagrangian dual maximum-likelihood relaxation: reinterpreting regularized lattice decoding," IEEE Transactions on Signal Processing, vol. 62, no. 2, pp. 511-524, 2014.

[23] D. Y. Gao, Duality Principles in Nonconvex Systems: Theory, Methods and Applications, vol. 39 of Nonconvex Optimization and its Applications, Springer, Berlin, Germany, 2000.

[24] D. Y. Gao, "Canonical duality theory and solutions to constrained nonconvex quadratic programming," Journal of Global Optimization, vol. 29, no. 4, pp. 377-399, 2004.

[25] D. Y. Gao, "Solutions and optimality criteria to box constrained nonconvex minimization problems," Journal of Industrial and Management Optimization, vol. 3, no. 2, pp. 293-304, 2007.

[26] D. Y. Gao, L. T. Watson, D. R. Easterling, W. I. Thacker, and S. C. Billups, "Solving the canonical dual of box- and integerconstrained nonconvex quadratic programs via a deterministic 
direct search algorithm," Optimization Methods \& Software, vol. 28, no. 2, pp. 313-326, 2013.

[27] V. Latorre and D. Y. Gao, "Canonical dual solutions to nonconvex radial basis neural network optimization problem," Neurocomputing, vol. 134, pp. 189-197, 2014.

[28] X. Zhou, D. Y. Gao, and C. Yang, “Canonical primal-dual algorithm for solving fourth-order polynomial minimization problems," Applied Mathematics and Computation, vol. 227, pp. 246255, 2014.

[29] S.-C. Fang, D. Y. Gao, R.-L. Sheu, and S.-Y. Wu, "Canonical dual approach to solving 0-1 quadratic programming problems," Journal of Industrial and Management Optimization, vol. 4, no. 1, pp. 125-142, 2008.

[30] Z. Wang, S.-C. Fang, D. Y. Gao, and W. Xing, "Global extremal conditions for multi-integer quadratic programming," Journal of Industrial and Management Optimization, vol. 4, no. 2, pp. 213-225, 2008.

[31] Z. Wang, S.-C. Fang, D. Y. Gao, and W. Xing, "Canonical dual approach to solving the maximum cut problem," Journal of Global Optimization, vol. 54, no. 1, pp. 341-351, 2012.

[32] M. Grant and S. Boyd, CVX: Matlab Software for Disciplined Programming, Version 1.2, 2010, http://cvxr.com/cvx/. 


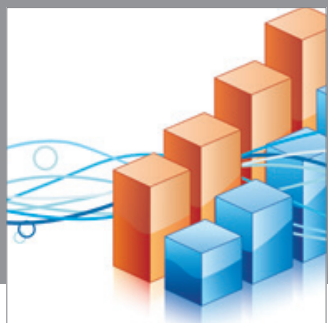

Advances in

Operations Research

mansans

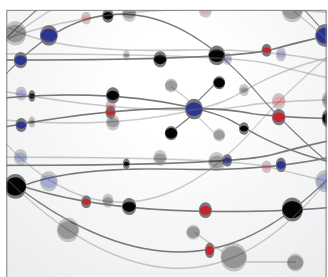

The Scientific World Journal
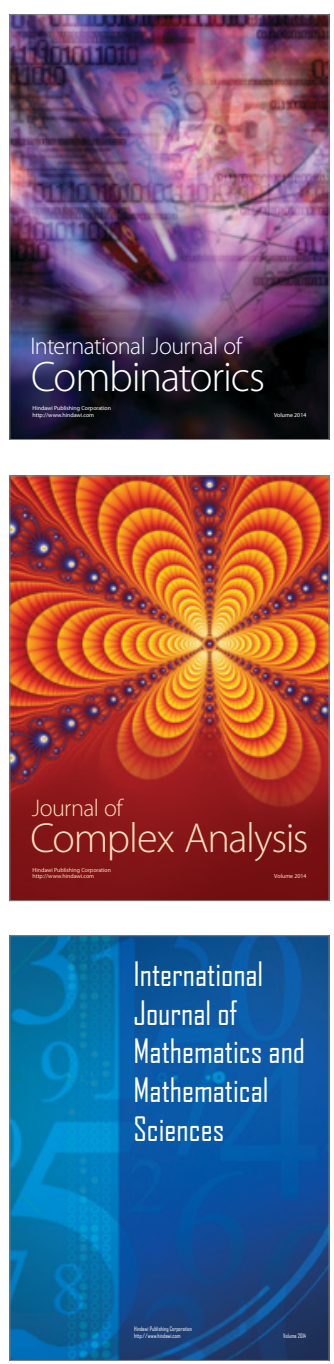
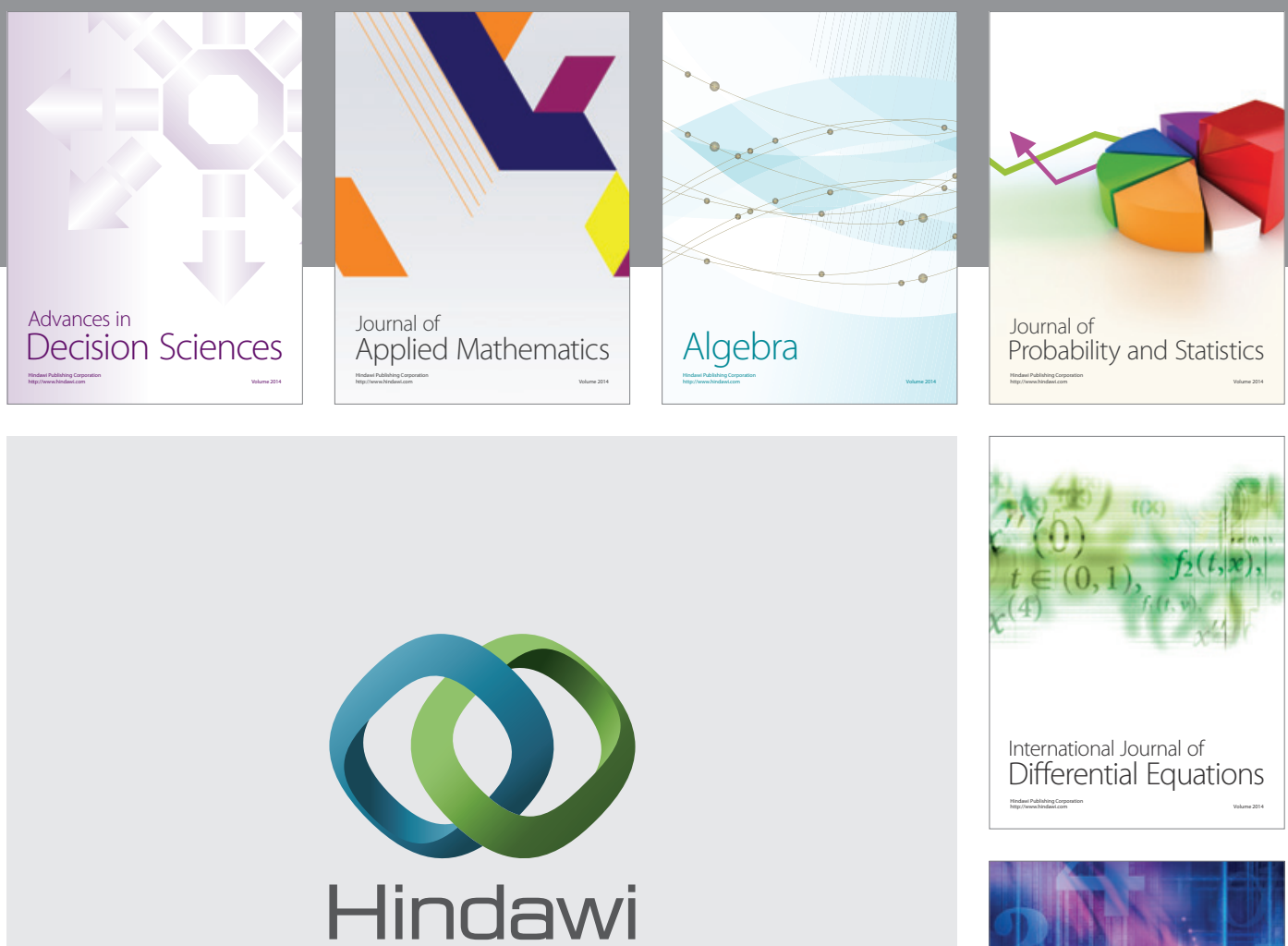

Submit your manuscripts at http://www.hindawi.com
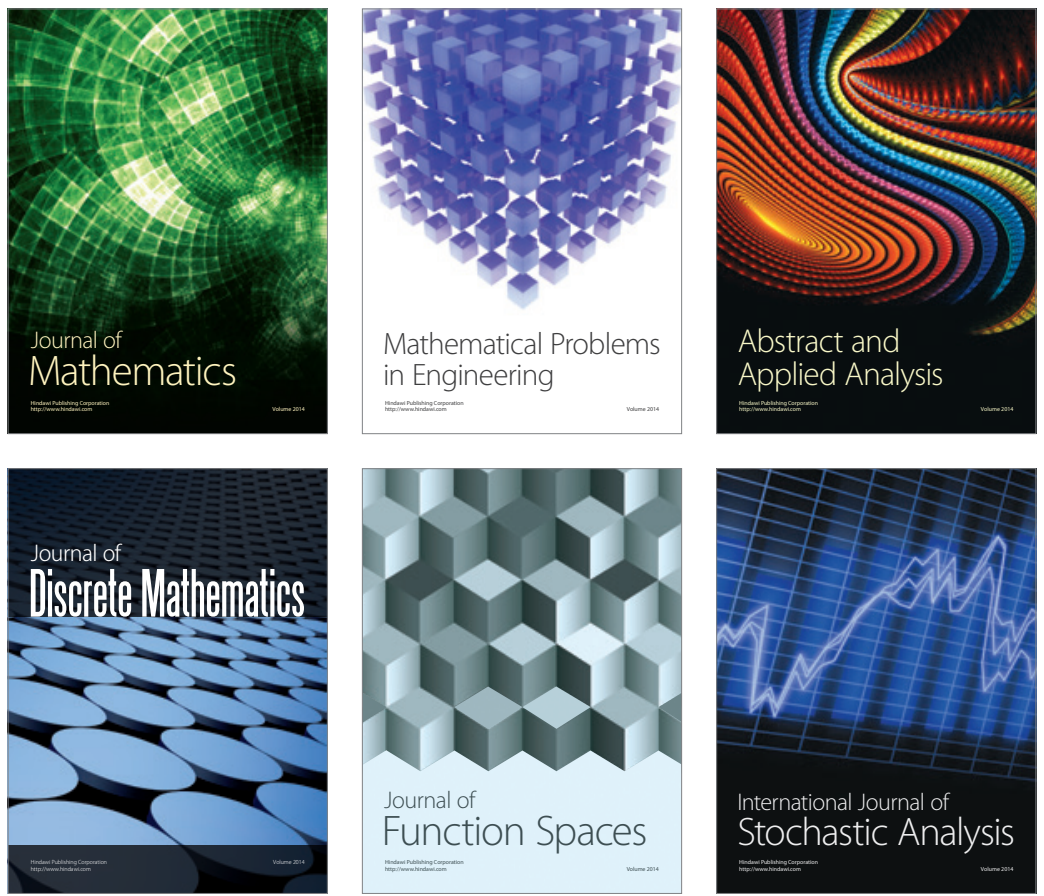

Journal of

Function Spaces

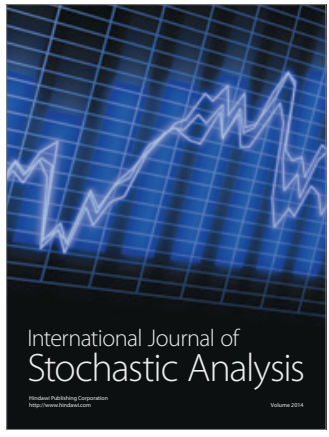

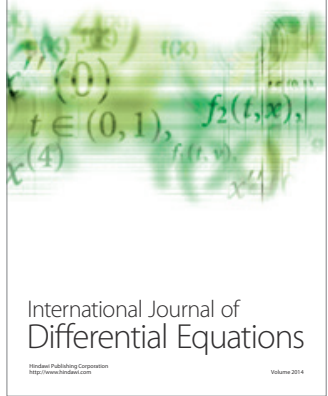
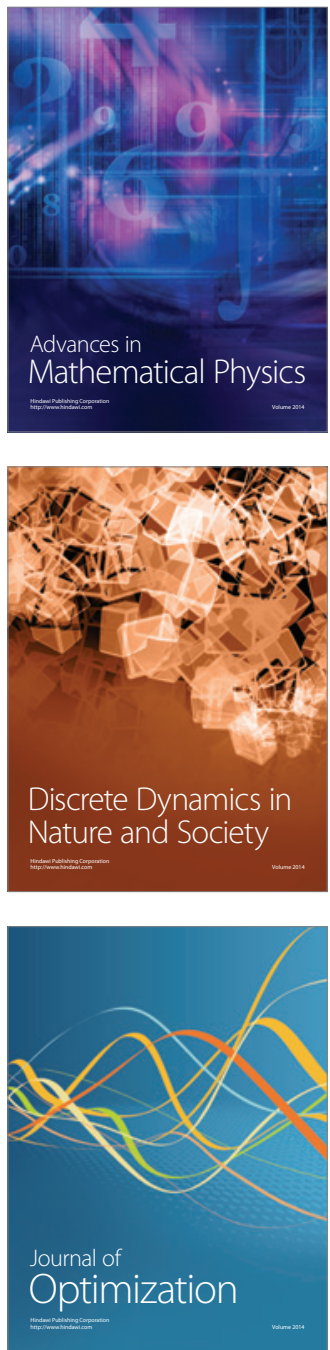